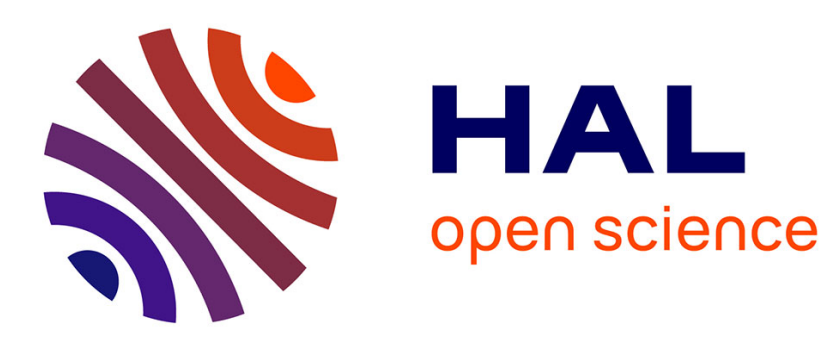

\title{
Asymptotic properties of infinite Leslie matrices
}

Frédéric Gosselin, J.D. Lebreton

\section{To cite this version:}

Frédéric Gosselin, J.D. Lebreton. Asymptotic properties of infinite Leslie matrices. Journal of Theoretical Biology, 2009, 256 (2), p. 157 - p. 163. 10.1016/j.jtbi.2008.09.018 . hal-00454506

\section{HAL Id: hal-00454506 https://hal.science/hal-00454506}

Submitted on 8 Feb 2010

HAL is a multi-disciplinary open access archive for the deposit and dissemination of scientific research documents, whether they are published or not. The documents may come from teaching and research institutions in France or abroad, or from public or private research centers.
L'archive ouverte pluridisciplinaire HAL, est destinée au dépôt et à la diffusion de documents scientifiques de niveau recherche, publiés ou non, émanant des établissements d'enseignement et de recherche français ou étrangers, des laboratoires publics ou privés. 


\title{
Asymptotic properties of infinite Leslie matrices
}

\author{
Frédéric GOSSELIN $^{\mathrm{a}, \mathrm{b}}$ \& Jean-Dominique LEBRETON ${ }^{\mathrm{a}, *}$ \\ ${ }^{a}$ Centre d'Ecologie Fonctionnelle et Evolutive, CNRS, C.E.F.E., 1919 Route de Mende, F- \\ 34293 Montpellier Cedex 5, France \\ ${ }^{\mathrm{b}}$ Cemagref, UR EFNO, F-45290 Nogent-sur-Vernisson, France \\ E-mail addesses: frederic.gosselin@ cemagref.fr, jean-dominique.lebreton@cefe.cnrs.fr.
}

Published in: Journal of Theoretical Biology (2009), vol. 256, $\mathrm{n}^{\circ} \mathbf{2}$, p. 157-163

doi:10.1016/.j.jtbi.2008.09.018

http://dx.doi.org/10.1016/i.jtbi.2008.09.018

\footnotetext{
* to whom all correspondence should be addressed (e-mail address: jean-dominique.lebreton@cefe.cnrs.fr, telephone number : +334676132 01; fax number: +33467412138).
} 


\begin{abstract}
The stable population theory is classically applicable to populations in which there is a maximum age after which individuals die. Demetrius (1972) extended this theory to infinite Leslie matrices, in which the longevity of individuals is potentially infinite. However, Demetrius had to assume that the survival probability per time step tends to 0 with age. We generalise here the conditions of application of the stable population theory to infinite Leslie matrix models and apply these results to two examples, including or not senescence.
\end{abstract}

Keywords: stable population theory; Leslie matrix; Usher matrix; senescence; infinite matrix. 


\section{Introduction}

The Leslie matrix is a classical model for population projection (Caswell, 2000), characterised by asymptotically exponential growth and by the convergence to a stationary age distribution, as a result of the spectral decomposition in the Perron-Frobenius theorem (e.g. Sykes, 1969; Seneta, 1981). The Leslie matrix model has been used in general under the assumption that all individuals die before a maximal age $n$. The projection matrix is then an $n \times n$ matrix. A less restrictive assumption leading to the same representation is that all individuals stop reproducing at age $n$, older individuals playing no role in population growth. However, in natural populations, the evidence for senescence is often scanty and controversial (Gaillard et al., 1994). This has led Usher (1972) to define an age $n$ at which demographic performances, i.e. both survival and fecundity, stabilise. Age class $n$ is then made up of individuals aged $n$ or more. The underlying population model can be represented either by an $n \times n$ matrix with a survival term in the lower corner of the matrix or by an infinite matrix (cf. example 1 in section 5).

Although a model similar to an infinite Leslie matrix model is frequently used in the framework of the stable population theory based on the Lotka equation (see e.g. Mertz, 1971), the mathematics of such a model have only been considered by Demetrius (1972). Infinite nonnegative irreducible matrices do not necessarily have the same properties as finite nonnegative irreducible matrices (e.g. Vere-Jones, 1967, 1968). To show that the classical Leslie matrix results hold for an infinite matrix, Demetrius used an analogy of the classical Perron-Frobenius theorem for infinite matrices (Krein and Rutman, 1948) under restrictive assumptions on the matrix coefficients. In particular, Demetrius' results do not apply to the infinite matrix form of the Usher model. Obviously, this leaves some hope of getting more general results for infinite Leslie matrices. The purpose of this paper is to provide such results, based on spectral properties of linear operators less stringent than those used by 
Demetrius. We also develop conditions under which various quantities of demographic interest are defined and provide illustrations of our results through two examples. The last example echoes a variety of studies that concentrated on evidence for a plateau in mortality or at least mortality deceleration with age (e.g. Pletcher \& Curtsinger, 1998; Vaupel et al. 1998; Partridge \& Mangel, 1999). However, to our knowledge, nobody ever considered how to produce population projections under such a mortality scheme. As convergence of the mortality (or equivalently, survival) probability to the plateau value is progressive, it is impossible to set up a model with a finite number of age classes, albeit using an ad hoc truncation. If an infinite matrix is used, the results by Demetrius (1972) do not apply since too restrictive. Example 2 covers this case and provides a clear rationale for finite dimensional truncation. The approach proposed makes it possible to easily implement in a reliable fashion any model with a mortality plateau reached asymptotically with age. We finally discuss the bearing of our results on the study of mortality deceleration and mortality plateaus (e.g. Pletcher and Curtsinger 1998; Vaupel et al. 1998).

\section{A broad generalisation of Demetrius' (1972) results}

Like Demetrius (1972), we denote as:

$a_{i}$ the net fecundity of a female aged $i$, i.e. the (average) number of females aged 1 at the next time step with a mother aged $i$, where $i$ is a positive integer;

$b_{i}$ the probability of survival of a female from age $i$ to age $i+1$.

The infinite Leslie matrix $M$ is then equal to: 


$$
M=\left[\begin{array}{cccccc}
a_{1} & a_{2} & \cdots & a_{i} & \cdots & \cdots \\
b_{1} & 0 & \cdots & 0 & \cdots & \\
0 & b_{2} & \ddots & \vdots & & \\
\vdots & 0 & \ddots & 0 & \ddots & \\
0 & \vdots & \ddots & b_{i} & \ddots & \ddots \\
\vdots & \vdots & \vdots & 0 & \ddots & \ddots
\end{array}\right]
$$

We denote as $M_{n}$ the $n \times n$ Leslie matrix obtained by truncating $M$ after age $n$ :

$$
M_{n}=\left[\begin{array}{ccccc}
a_{1} & a_{2} & \cdots & a_{n-1} & a_{n} \\
b_{1} & 0 & \cdots & 0 & 0 \\
0 & b_{2} & \cdots & \vdots & \vdots \\
\vdots & \ddots & \ddots & 0 & \vdots \\
0 & \cdots & 0 & b_{n-1} & 0
\end{array}\right]
$$

We also denote as $r\left(M_{n}\right)$ the spectral radius of the matrix $M_{n}$, i.e. the maximum modulus of the eigenvalues.

As Demetrius (1972), we assume $0 \leq a_{i}<\infty$ and $0<b_{i} \leq 1$ for every $i$. We also assume throughout that the greatest common divisor of the indices $i$ of the positive $a_{i}$ equals 1 , which we think is a biologically unrestrictive assumption.

Demetrius (1972) demonstrated that the matrix $M$ behaves as a finite Leslie matrix under the following assumptions :

(i) $a_{i}>0$ for infinitely many $i$ and the sequence $\left(a_{i}\right)_{i}$ is bounded from above (i.e. $\left.\sup _{i} a_{i}<\infty\right)$, and

and (ii) $\lim _{i \rightarrow \infty} b_{i}=0$.

The latter condition appears as quite restrictive, and prevents to use these results when mortality stabilises to a positive plateau with increasing age. More general sufficient conditions (Appendix 1) are as follows. If: 
(i) $a_{i}>0$ for infinitely many $i$ and the sequence $\left(a_{i}\right)_{i}$ is bounded from above (i.e. $\left.\sup _{i} a_{i}<\infty\right)$, and

and (iv) there is $n$ such that $\lim \sup b_{i}<r\left(M_{n}\right)$, $i \rightarrow \infty$

then the operator associated with the matrix $M$ asymptotically behaves as a finite Leslie matrix (Theorem 1 in Appendix 1).

The assumption (iv) is more general than (ii) and brings a marked improvement. Indeed, it makes the standard results of stable population theory valid in particular for the following infinite Leslie matrices :

- the infinite counterpart of the Usher matrix (example 1 in Section 5);

and - populations whose survival probabilities converge to a plateau under mild conditions (example 2 in section 5).

It should also apply to most demographic schemes whether they include a mortality deceleration or not (Carey et al. 1992; Mueller \& Rose, 1996; Pletcher and Curtsinger 1998; Vaupel et al. 1998; Rose et al. 2002; Steinsaltz 2005; Styer et al 2007). Actually, condition (iv) is obviously satisfied if for some $n$, the matrix $M_{n}$ has a leading eigenvalue (i.e. spectral radius) $r\left(M_{n}\right)$ greater than 1 . If on the contrary $\sup _{n} r\left(M_{n}\right) \leq 1$, condition (iv) is met if $b_{i}$ remains less than $r\left(M_{n}\right)-\varepsilon$ for some $n$ and $\varepsilon>0$ and for all but a finite number of $i$, a condition much more general than (ii). 


\section{Demographic quantities of interest}

Asymptotic exponential growth, stable age distribution, reproductive values

Under conditions (i) and (iv), Eq. (A1.1) in Appendix 1 tells us that the matrix $M$ has asymptotic properties similar to those of finite Leslie matrices (Caswell, 2000). Indeed, when time $\theta$ tends to infinity:

$$
M^{\theta} x \approx r(M)^{\theta}(u, x) v
$$

where $\approx$ means asymptotic equivalence in $l_{1}\left(F_{k}\right)$ norm for every $k>0$ (cf. notation in Appendix 1). This signifies that for every $k>0$ and $x=\left(x_{i}\right)_{i=1,2, \ldots}$ such that $\sum_{i=1}^{\infty} i^{k}\left|x_{i}\right|<\infty, \sum_{i=1}^{\infty} i^{k}\left|\left(M^{\theta} x\right)_{i}-r(M)^{\theta}(u, x) v_{i}\right|$ converges to 0 when time $\theta$ tends to infinity. In Demetrius (1974)'s words, this means that under this norm, M is strongly ergodic. In other words, our results show that under our conditions, by restricting the vector space on which the matrix $\mathrm{M}$ acts - for some $k>0$, to those vectors $x=\left(x_{i}\right)_{i=1,2, \ldots}$ such that $\sum_{i=1}^{\infty} i^{k}\left|x_{i}\right|<\infty-M$ has asymptotic properties similar to those of finite Leslie matrices. The vector $v=\left(v_{j}\right)_{j}$, with an infinite number of components, gives the stable age distribution. Similarly, the vector $u=\left(u_{j}\right)_{j}$ gives the reproductive value of age classes $j$. These two infinite vectors are such that for every $k>0, \sum_{i=1}^{\infty} i^{k}\left|v_{i}\right|<\infty$ and $\sup _{i=1,2, \ldots} \frac{\left|u_{i}\right|}{i^{k}}<\infty$ (Appendix 1). 
The speed of the convergence of $\frac{M^{\theta} x}{r(M)^{\theta}}$ to $(u, x) v$ is then asymptotically described by the damping ratio $\rho=\frac{r(M)}{r(S)}$ (cf. notation in Appendix 1): the bigger this ratio, the quicker the convergence asymptotically.

\section{Relationship with stable population theory}

Is there an equivalent of the characteristic equation in infinite Leslie matrices? We are not aware of any generalisation of the characteristic equation method for infinite matrices. This constitutes another evidence of the difference between the spectral theory of infinite matrices and that of finite matrices. We show in Appendix 2 that such an equation:

$$
\Phi(\lambda)=\sum_{i=1}^{\infty} \ell_{i} a_{i} \lambda^{-i}=1
$$

permits to determine the leading eigenvalue $\lambda=r(M)$ of an infinite Leslie matrix satisfying (i) and (iv), where $\ell_{i}=\prod_{i=1}^{i-1} b_{i}$, with $\ell_{1}=1$. Following Murray (1991), we call Eq. (2) the discrete time version of the Lotka equation.

Even if Eq. (2) has a priori no bearing on eigenvalues of $M$ other than the dominant one, it resembles the characteristic polynomial of finite matrices. Based on it, one obtains, as classically for finite Leslie matrices (e.g. Caswell, 2000, or Lebreton, 1996): $u_{i}=\lambda^{-i} u_{1} \sum_{j=i}^{\infty} \ell_{j} a_{j} \lambda^{-j}$ and $v_{i}=\ell_{i} \lambda^{-(i-1)} v_{1}$, for every $i \geq 2$, where $u$ and $v$ are the vectors defined in Theorem 1 (cf. Appendix 1). 
We prove in Appendix 2 that, under our conditions (i) and (iv), we can differentiate the function $\Phi$ in a neighbourhood of $\lambda=r(M)$ :

$$
\Phi^{\prime}(\lambda)=\sum_{i=1}^{\infty}-i \ell_{i} a_{i} \lambda^{-(i+1)}<\infty
$$

Denoting $T=\sum_{i=1}^{\infty} i \ell_{i} a_{i} \lambda^{-i}$, we get $\Phi^{\prime}(\lambda)=-\frac{T}{\lambda}$. The quantity $T$ can be written as $T=\sum_{i=1}^{\infty} i \frac{v_{i}}{\lambda v_{1}} a_{i}$, with $T<\infty$, as a result of $\sum_{i=1}^{\infty} A^{i} v_{i}<\infty$ and (i), where A is any number such that $A>1$ and $\limsup A b_{i}<r\left(M_{n}\right)$ (cf. Appendix 2). As usual (e.g. Houllier and $i \rightarrow \infty$

Lebreton, 1986), $T$ is called the generation time and can be interpreted as the mean age of mothers at birth once the stable age distribution has been reached.

The lifelong contribution of an individual, $R_{0}=\sum_{i=1}^{\infty} a_{i} \ell_{i}$, or net reproductive value, is obviously finite under (i) and $\limsup _{i \rightarrow \infty} b_{i}<1$, since $\ell_{i}=\prod_{i=1}^{i-1} b_{i}$.

\section{Sensitivity of population parameters to variation in individual parameters}

Finally, under further conditions, unlikely to be limiting for infinite Leslie matrices, sensitivity analysis results identical to those in finite dimension (e.g. Caswell, 2000, Chapter 9) are available (Gosselin, 1997, Submitted). For instance, using the terminology in Demetrius (1989), the sensitivity of the population-level dominant eigenvalue $r(M)$ concomitant to the variation in the individual demographic parameter $d m_{i, j}$ of the $(i, j)$ entry of matrix $M$ is equal to $\frac{\mathrm{d} r(M)}{\mathrm{d} m_{i, j}}=\frac{u_{i} v_{j}}{(u, v)}$. Another example is when all fecundities (resp. 
survivals) vary proportionally: the sensitivity of the dominant eigenvalue $r(M)$ to such changes is $\frac{u_{1} \sum_{j=1}^{\infty} a_{j} v_{j}}{(u, v)}$ (resp. $\left.\frac{\sum_{j=1}^{\infty} b_{j} u_{j+1} v_{j}}{(u, v)}\right)$. A final application is the situation when there is senescence - i.e. no reproduction above a maximum age bound - and survival rates are disturbed only above the senescence age: the abovementioned results show that the asymptotic growth rate $r(M)$ remains constant under such perturbations, an infinite dimensional echo of evolutionary considerations on the non-selection of genes that would have an impact only on survival after senescence (e.g. Pletcher \& Curtsinger, 1998; Partridge \& Mangel, 1999). The question is then to determine whether such perturbation schemes are biologically and evolutionarily realistic (Pletcher \& Curtsinger, 1998; Partridge \& Mangel, 1999).

\section{Convergence of the dominant value of finite truncations of the infinite Leslie matrix}

While suppressing a maximum age bound may have a strong effect on the model results (e.g. Lande, 1988), the numerical manipulation of infinite Leslie matrices, e.g. for calculating the asymptotic growth rate, will require to reintroduce some kind of maximum age bound at the computer implementation stage. Then, under general conditions which are met under the conditions of Theorem 1 in Appendix 1, $\lim _{n \rightarrow \infty} r\left(M_{n}\right)=r(M)$ (cf. Seneta, 1981), i.e. the eigenvalue of the $n \times n$ truncated matrix converges to the infinite matrix leading eigenvalue when $\mathrm{n}$ tends to infinity. Once a good approximation $r\left(M_{n}\right)$ of $\lambda=r(M)$ has been obtained - as e.g. in Example 2 of section 5 -, the corresponding eigenvectors $u$ and $v$ can be easily calculated from the formulas in section 3. Further results can be found in Gosselin (1997, Appendix 7). 


\section{Some examples}

\section{Example 1}

The Usher model with stabilisation at age $n$ of net fecundity (with value $a>0$ ) and of survival (with value $b>0$ ) has both a finite matrix form, i.e.

$$
M^{\prime}=\left[\begin{array}{ccccc}
a_{1} & a_{2} & \ldots & a_{n-1} & a \\
b_{1} & 0 & \ldots & 0 & 0 \\
0 & b_{2} & \ddots & \vdots & \vdots \\
\vdots & \ddots & \ddots & 0 & 0 \\
0 & \ldots & 0 & b_{n-1} & b
\end{array}\right],
$$

and an infinite matrix form, i.e.

$$
M=\left[\begin{array}{ccccccc}
a_{1} & \cdots & a_{n-1} & a & a & \cdots & \cdots \\
b_{1} & 0 \cdots & 0 & 0 & 0 & \cdots & \cdots \\
0 & \ddots & \ddots & \vdots & \vdots & & \\
\vdots & \ddots & b_{n-1} & 0 & \ddots & & \\
0 & \ddots & 0 & b & 0 & & \vdots \\
\vdots & \vdots & \vdots & 0 & b & \ddots & \\
\vdots & \vdots & \vdots & \vdots & \ddots & \ddots & \ddots
\end{array}\right]
$$

We here consider this last representation. $M$ obviously satisfies (i). The spectral radius of the truncated matrices $M_{N}$ (for $\left.N>n\right), \lambda_{N}=r\left(M_{N}\right)$, increases with $N$ and we denote $b^{\prime}=\sup _{N} \lambda_{N}$. We then have $b^{\prime}>b$, as can be proved from the Lotka equation associated with $M_{N}$ :

$$
\sum_{i=1}^{n-1} b_{1} b_{2} \ldots b_{i-1} a_{i} \lambda_{N}{ }^{-i}+\sum_{i=n}^{N-1} b_{1} b_{2} \ldots b_{n-1} b^{i-n} a \lambda_{N}{ }^{-i}=1
$$

which implies :

$$
\sum_{i=n}^{N-1} b_{1} b_{2} \ldots b_{n-1} b^{i-n} a \lambda_{N}^{-i}=\frac{b_{1} b_{2} \ldots b_{n-1} a}{b^{n}} \sum_{i=n}^{N-1} \frac{b^{i}}{\lambda_{N}{ }^{i}} \leq 1 \text {. }
$$


If $b^{\prime} \leq b$, the term $\sum_{i=n}^{N-1} \frac{b^{i}}{\lambda_{N}{ }^{i}}$ would obviously tend to infinity with $N$, and Eq. (3) would not be met for every $N$. Hence $b^{\prime}>b$, and condition (iv) holds for some $N$. From Theorem 1 in Appendix 1 and section 3, we get, for any $k>0$, that when time $\theta$ tends to infinity:

$$
M^{\theta} x \approx r(M)^{\theta}(u, x) v
$$

where $\approx$ means asymptotic equivalence in $l_{1}\left(F_{k}\right)$ norm; the generation time, $T$, and the lifelong contribution of an individual, $R_{0}$, are well defined (provided $b<1$ for $R_{0}$ ). Since these quantities are not simpler than above, we refer the reader to section 3 for the expressions of these quantities.

\section{Example 2}

Infinite matrix models are relevant in all cases where survival varies with age in a complex fashion, in particular when there is a senescent decrease in survival. Most empirical studies consider a simple function of age such as the inverse logit of a linear or quadratic function of age. The survival will then decrease to 0, and Demetrius (1972) result on infinite matrices is sufficient to handle this case. However, it may be more realistic to consider that survival decreases to a plateau (Pletcher and Curtsinger 1998; Vaupel, et al. 1998; Steinsaltz 2005). Let us consider for instance an example in which the annual survival probability, $b_{i}$ varies between 0.6 and 1 according to the inverse logit of a quadratic function of age, denoted as $i$. Survival increases from prime age to mature females, and then decreases progressively to reach a senescent plateau (Figure 1).

As survival reaches the senescent plateau value 0.6 only asymptotically, the population matrix model is necessarily infinite. Les us assume moreover that reproduction starts at age 2 , with a net fecundity equal to 0.35 female aged one per female aged 2 or more. A version of the model truncated at age $\mathrm{n}$ either considers that all individuals die at age $\mathrm{n}$ (Leslie matrix) or 
that survival stabilizes at its current value (Usher matrix). The Leslie $M_{5}$ and Usher $U_{5}$ matrices for $\mathrm{n}=5$ are given below:

$$
M_{5}=\left[\begin{array}{ccccc}
0 & 0.35 & 0.35 & 0.35 & 0.35 \\
0.87 & 0 & 0 & 0 & 0 \\
0 & 0.88 & 0 & 0 & 0 \\
0 & 0 & 0.89 & 0 & 0 \\
0 & 0 & 0 & 0.90 & 0
\end{array}\right], U_{5}=\left[\begin{array}{ccccc}
0 & 0.35 & 0.35 & 0.35 & 0.35 \\
0.87 & 0 & 0 & 0 & 0 \\
0 & 0.88 & 0 & 0 & 0 \\
0 & 0 & 0.89 & 0 & 0 \\
0 & 0 & 0 & 0.90 & 0.90
\end{array}\right]
$$

The corresponding dominant eigenvalues are 1.0073 and 1.1582 , respectively. Since with the notation of section 2, we have limsup $b_{i}=0.6<r\left(M_{5}\right)=1.0073$, condition (iv) holds. Our $i \rightarrow \infty$

results ensure that the dominant eigenvalues of the two types of matrices converge to that of the infinite matrix when the order of truncation increases. The result is straightforward for matrix $M_{n}$, based on Section 4. The result also holds for matrix $U_{n}$, based on a slightly different proof on the same lines (unpublished results). This convergence is illustrated in Figure 2. At order 43 the two eigenvalues are numerically indistinguishable up to 9 decimals, and equal to 1.163342945 .

\section{Discussion}

Our work was motivated by the possibility of a discontinuity between the asymptotic behaviour of finite, truncated matrix models - including for example a maximum age or assuming a constant survival rate above a given age bound-, and their infinite counterpart. Our results show that this is not the case. They indeed prove that the stable population theory still holds with an infinite number of age classes under specific conditions (points (i) and (iv) in section 2). These conditions are much more general than those proposed by Demetrius (1972) as they do not require that the age-specific survival probability tends to 0 with age. In particular, they are met by the infinite matrix version of the Usher matrix. Our approach also 
provides a reliable way of assessing the effect of truncation of an infinite matrix model on the population growth rate. Our results show that the population quantities involved in the stable age theory are defined in a consistent fashion in the infinite matrix formulation (section 3 ) and are the limits of the same quantities obtained under finite truncation (section 4). Similar results may also be obtained for stage-classified population matrices (Caswell, 2000) with an infinite number of stages. However, due to the more complex shape of the matrices, specific checks and conditions have to be developed for each case.

We could not completely match our results with those of Demetrius (1983; Theorems 5.0 and 5.1). Under the conditions $a_{1}=0, a_{i} \equiv a>0, i \geq 2$, and $\Phi(1)=\sum_{i=1}^{\infty} \ell_{i} a_{i}>1$, Theorem 5.0 in Demetrius (1983) shows the existence of a unique equilibrium state. It can actually be shown that the conditions of Theorem 5.0 in Demetrius (1983) imply our conditions (i) and (iv) (cf. the proof of Theorem 5.0 in Demetrius 1983): this theorem must therefore be considered as a special case of our Theorem 1 in Appendix 1 . The reverse holds when $a_{1}=0$, $a_{i} \equiv a>0, i \geq 2$, and $\Phi(1)=\sum_{i=1}^{\infty} \ell_{i} a_{i}=1:$ then, Theorem 1 in Appendix 1 is a special case of Theorem 5.1, (1), in Demetrius (1983). However, the results of both theorems are then surprisingly different, since our results imply - as Feller (1968, p. 330-331) - a unique equilibrium state which is iterparous in the terms of Demetrius (1983, p.734), whereas Demetrius (1983, Theorem 5.1, (1)) gets two equilibria, one iterparous, one semelparous. Actually, what is most striking with the results of Demetrius (1983), is the different number of equilibrium states given by his two theorems when applied to a matrix 


$$
M=\left[\begin{array}{cccccc}
0 & a & \cdots & a & \cdots & \cdots \\
b_{1} & 0 & \cdots & 0 & \cdots & \\
0 & b_{2} & \ddots & \vdots & & \\
\vdots & 0 & \ddots & 0 & \ddots & \\
0 & \vdots & \ddots & b_{i} & \ddots & \ddots \\
\vdots & \vdots & \vdots & 0 & \ddots & \ddots
\end{array}\right]
$$

such that $\limsup _{i \rightarrow \infty} b_{i}=r<1$ and $\Phi(1)=\sum_{i=1}^{\infty} \ell_{i} a_{i}=1-$ then his Theorem 5.1, (1) applies and yields two equilibria - whereas when considering the matrix $\frac{M}{r^{\prime}}$, with $r<r^{\prime}<1$, Theorem 5.0 of Demetrius (1983) applies and yields only one equilibrium.

The two examples presented in section 5 cover population models in presence of a mortality plateau, whether it is reached at a finite age (example 1) or only asymptotically with increasing age (example 2). Our results would therefore make possible a general, populationlevel treatment of the continuum from negligible senescence (Finch, 1998; Finch \& Austad, 2001) to senescence, including the intermediate case of mortality deceleration and mortality plateaus: Example 2 in section 5 treats the case of mortality plateaus without a maximum age bound; it would similarly be possible to handle cases where fecundity decreases with age without a maximum reproductive age. We expect these results will encourage the use of realistic survival variation with age of the type considered here.

Our main result - Theorem 1 in Appendix 1 - may be generalised to multisite Leslie matrices (Lebreton, 1996) or to frailty models, incorporating a finite number of demographic profiles (e.g. Vaupel \& Yashin, 1985; Vaupel \& Carey, 1993), with an infinite number of age classes. Then, the same kind of results as in section 3 and appendices 1 and 2 apply if we consider, instead of conditions (i) and (iv), the following two conditions:

(i') the sequence $\left(\left\|A_{i}\right\|\right)_{i}$ is bounded (i.e. $\left.\sup _{i}\left\|A_{i}\right\|<\infty\right)$, and 
(iv') there is $n$ such that $\lim \sup \left\|B_{i}\right\|<r\left(M_{n}\right)$,

$$
i \rightarrow \infty
$$

where $A_{i}$ and $B_{i}$ are $s \times s$ matrices corresponding respectively to the reproduction and dispersal of new-borns from individuals aged $i$ and to the survival- and dispersal in the case of multisite models - of individuals aged $i$, where $s$ is the finite number of sites or frailty groups. This could encourage the population-level evolutionary analysis of two of the hypotheses identified by Vaupel et al. (1998) for mortality deceleration: i.e. mortality correlation and heterogeneity in frailty, with various levels of heritability in frailty (e.g. Ducrocq et al. 2000). Generalisations to an infinite number of sites or frailty groups would require more specific approaches. Similarly, the incorporation of dependences between individuals, the history of individuals or individual-environment interactions - other potential classes of explanations for mortality deceleration proposed by Vaupel et al. (1998) and Partridge \& Mangel (1999) - would require the use of Markov chain models (Gosselin \& Lebreton 2000; Gosselin, 2001; Lebreton et al. 2007) or more complicated stochastic models. The inclusion of an infinite number of age classes in these models would also require further developments.

\section{Appendix 1. Spectral decomposition of the infinite Leslie matrix model}

For the sake of simplicity, we use in this paper the same notation for a matrix and for the linear operator associated with it. A linear operator $U$ on the Banach space $E$ is compact if the image by $U$ of every bounded subset of $E$ is relatively compact, i.e. for every infinite bounded sequence $\left(x_{n}\right)$ of elements in $E$ (i.e. there is $M>0$ such that for every $n$, 
$\left.\left\|x_{n}\right\|_{E}<M\right)$, we get by $U$ an image $\left(U x_{n}\right)$ from which we can extract a convergent subsequence, i.e. there are a sequence of integers $\left(k_{n}\right)$ and $y$ in $E$ such that

$$
\lim _{n \rightarrow \infty} k_{n}=\infty \text { and } \lim _{n \rightarrow \infty}\left\|U x_{k_{n}}-y\right\|_{E}=0
$$

A linear operator $U$ is quasi-compact if there exists a positive integer $n$ and a linear operator $V$ on $E$ such that $U^{n}-V$ is a compact operator and $r_{E}(V)<r_{E}(U)^{n}$, where $r_{E}($.$) denotes the$ spectral radius of a linear operator. The interest of these notions is that spectral properties of compact or quasi-compact operators make them (spectrally) closer to operators in finite dimensional Banach spaces than non-compact operators, whose spectrum can be continuous.

Demetrius (1972) showed that under assumptions (i) and (ii) in section 2, the matrix $M$ corresponds to a bounded and compact operator on the Hilbert space $l_{2}$ made of the complex sequences $\left(x_{i}\right)_{i}$ such that $\sum_{i}\left|x_{i}\right|^{2}<\infty$. Demetrius (1974) also mainly relied on the notion of compactness to develop sufficient conditions for different kinds of ergodicity. Then, from Krein and Rutman (1948), the operator $M$ behaves asymptotically as a classical finite Leslie matrix. We obtain more general sufficient conditions than Demetrius (1972) based on the notion of quasi-compactness (Sasser, 1964) rather than compactness, on other Banach spaces than $l_{2}$, namely, as in Vere-Jones (1968) and Gosselin (2001):

$$
l_{\infty}(F)=\left\{\left(x_{j}\right)_{j} ;\left\|\left(x_{j}\right)_{j}\right\|_{\infty, F}:=\sup _{j} \frac{\left|x_{j}\right|}{F(j)}<\infty\right\},
$$

And

$$
l_{1}(F)=\left\{\left(y_{j}\right)_{j} ;\left\|\left(y_{j}\right)_{j}\right\|_{1, F}:=\sum_{j} F(j)\left|y_{j}\right|<\infty\right\},
$$


where $F$ is a map on positive integers $j$ such that $F(j)>0$ and $\lim _{j \rightarrow \infty} F(j)=\infty . l_{\infty}(F)$ is the dual space of $l_{1}(F)$. In brief, quasi-compactness corresponds to a weaker decrease in the terms in $M$ with their row and column number than compactness, and nevertheless implies that $M$ behaves in certain respects as a finite matrix.

Theorem 1. Let $M$ be the matrix defined above. Further assume:

(i) $a_{i}>0$ for infinitely many $i$ and the sequence $\left(a_{i}\right)_{i}$ is bounded from above (i.e. $\left.\sup _{i} a_{i}<\infty\right)$, and

(iv) there is $n$ such that $\lim \sup b_{i}<r\left(M_{n}\right)$.

$$
i \rightarrow \infty
$$

Then, for every $k>0$, the operator associated with the matrix $M$ on the Banach space $l_{1}\left(F_{k}\right)$ is quasi-compact, where $F_{k}(i)=i^{k}$, and

$$
M=r(M) v^{\mathrm{t}} u+S,
$$

where

$r(M)$, the spectral radius of the operator $M$ on $l_{1}\left(F_{k}\right)$, is real positive;

$S$ is a bounded operator on $l_{1}\left(F_{k}\right)$ with spectral radius $r(S)$ less than $r(M)$; and $u$ and $v$ are positive vectors in $l_{\infty}\left(F_{k}\right)$ and $l_{1}\left(F_{k}\right)$ respectively, such that $(u, v)={ }^{\mathrm{t}} u v=\sum_{i} u_{i} v_{i}=1, S v=0$ and ${ }^{\mathrm{t}} S u=0,{ }^{\mathrm{t}} u$ (resp. ${ }^{\mathrm{t}} S$ ) denoting the transposed vector (resp. matrix) of $u$ (resp. S). $u$ and $v$ are thus eigenvectors of the matrices ${ }^{t} M$ and $M$, respectively, associated with the eigenvalue $r(M)$. 
Proof. The transposed matrix of $M$, denoted by ${ }^{\mathrm{t}} M$, is:

$$
{ }^{t} M=\left[\begin{array}{cccccc}
a_{1} & b_{1} & 0 & \cdots & 0 & \cdots \\
a_{2} & 0 & b_{2} & 0 & \cdots & \\
\vdots & \vdots & \ddots & \ddots & \ddots & \vdots \\
a_{i} & 0 & \cdots & 0 & b_{i} & 0 \\
\vdots & \vdots & \ddots & \ddots & 0 & \ddots
\end{array}\right]
$$

From (i) and (iv), the operator ${ }^{\mathrm{t}} M$ on $l_{\infty}\left(F_{k}\right)$ is bounded. From proposition 5.3 in Gosselin (2001), ${ }^{\mathrm{t}} M \quad$ is quasi-compact on $l_{\infty}\left(F_{k}\right)$ provided $\quad r(M)>0 \quad$ and $\limsup _{i \rightarrow \infty}\left[\frac{a_{i}}{i^{k}}+\frac{(i+1)^{k}}{i^{k}} b_{i}\right]<r(M)$. The first condition, $r(M)>0$, results from the aperiodicity of $M$. As to the second condition, $M \geq M_{n}$ implies $r(M) \geq r\left(M_{n}\right)$ for every $n$ (e.g. Seneta, 1981). Furthermore, $\sup _{i} a_{i}<\infty$ implies $\lim _{i \rightarrow \infty} \frac{a_{i}}{{ }^{k}}=0$ for every $k>0$ and the second condition reduces to $\limsup _{i \rightarrow \infty} b_{i}<r(M)$. Hence, under (i) and (iv), the operators ${ }^{\mathrm{t}} M$ on $l_{\infty}\left(F_{k}\right)$ and $M$ on $l_{1}\left(F_{k}\right)$ are quasi-compact. Equation (A1.1) is then a direct application of lemma 6.1 in Gosselin (2001).

\section{Appendix 2. Relationship with stable population theory}

Some properties, trivial in the finite-dimensional case, raise the need of sound proofs in the infinite-dimensional case. Such is the case for the existence of a solution for the discrete time version of the infinite Lotka equation. The conditions and techniques we used in Appendix 1 actually make it possible to prove that $\lambda=r(M)$ fulfils the discrete time version of the Lotka equation: $\Phi(\lambda)=\sum_{i=1}^{\infty} \ell_{i} a_{i} \lambda^{-i}=1$. Although this point is treated by Feller (1968, p. 330- 
331 , when $\Phi(1) \geq 1$, the existence of $\lambda$ satisfying $\Phi(\lambda)=1$ is not obvious when $\Phi(1)<1$, since one can have $\Phi(1)<1$ and $\Phi\left(1^{-}\right)=\infty$. This is why Feller (1966, p. 360-363) assumed that such a $\lambda$ exists to find results when $\Phi(1)<1$.

Theorem 2. Let $M$ be the matrix defined above. Further assume (i) and (iv). Then, denoting $\lambda=r(M)$, we have: $\Phi(\lambda)=\sum_{i=1}^{\infty} \ell_{i} a_{i} \lambda^{-i}=1$ and $\Phi$ is differentiable in a neighbourhood of $\lambda$ with $\Phi^{\prime}(\lambda)=\sum_{i=1}^{\infty}-i \ell_{i} a_{i} \lambda^{-(i+1)}<\infty$.

Proof. We denote by $N_{1}(\theta)$ the population size in the first age class at time $\theta$ when the initial composition of the population is such that $N_{1}(0)=1$ and $N_{i}(0)=0, i \geq 2$. We also denote $\lambda=r(M), \lambda^{\prime}=r(S)$ and $\lambda_{n}=r\left(M_{n}\right)$ (cf. Appendix 1). We have already seen in the proof of Theorem 1 in Appendix 1 that $\lambda \geq \lambda_{n}$. Now, $\lambda_{n}$ satisfies the characteristic equation of the matrix $M_{n}$ :

$$
\sum_{i=1}^{n-1} \ell_{i} a_{i} \lambda_{n}^{-i}=1
$$

where $\ell_{i}=\prod_{i=1}^{i-1} b_{i}$, with $\ell_{1}=1$. This implies, together with $\lambda \geq \lambda_{n}$, that for every $n$ :

$$
\sum_{i=1}^{n-1} \ell_{i} a_{i} \lambda^{-i} \leq 1
$$

Whence,

$$
\sum_{i=1}^{\infty} \ell_{i} a_{i} \lambda^{-i} \leq 1
$$

Besides, for every $\varepsilon>0$ chosen such that $\lambda^{\prime}+\varepsilon<\lambda$, Theorem 1 implies

$$
N_{1}(\theta)=\lambda^{\theta} K v_{1}+o\left(\left(\lambda^{\prime}+\varepsilon\right)^{\theta}\right)
$$


where $o\left(\left(\lambda^{\prime}+\varepsilon\right)^{\theta}\right)$ is such that $\lim _{\theta \rightarrow \infty} \frac{\left\|o\left(\left(\lambda^{\prime}+\varepsilon\right)^{\theta}\right)\right\|_{1, F_{k}}}{\left(\lambda^{\prime}+\mathcal{E}\right)^{\theta}}=0$ for $k$ as in Theorem 1. But, as in e.g. Lebreton (1996), the very nature of the Leslie matrix model implies the following renewal equation:

$$
N_{1}(\theta)=\sum_{i=1}^{\theta} \ell_{i} a_{i} N_{1}(\theta-i), \text { for every } \theta
$$

These elements combine to prove that $\lambda$ satisfies:

$$
\Phi(\lambda)=\sum_{i=1}^{\infty} \ell_{i} a_{i} \lambda^{-i}=1
$$

We now prove formally that we can differentiate the function $\Phi$ in a neighbourhood of $\lambda$. Indeed, considering $A>1$ such that $\lim \sup A b_{i}<r\left(M_{n}\right)$, we can prove the same $i \rightarrow \infty$

results as in Theorem 1 , but on the Banach space $l_{1}\left(A^{F_{1}}\right)$, where $A^{F_{1}}(i)=A^{i}$. Indeed, following the same line as in the proof of Theorem 1, it is sufficient to show that $\limsup _{i \rightarrow \infty}\left[\frac{a_{i}}{A^{i}}+\frac{A^{i+1}}{A^{i}} b_{i}\right]<r(M)$, which follows directly from conditions (i) and (iv) and the condition met by $A$. Since the Leslie matrix model with a given initial population vector $N(0)$ has its own asymptotic behaviour, we know by taking $N(0)$ in the intersection of $l_{1}\left(F_{k}\right)$ and $l_{1}\left(A^{F_{1}}\right)$ that the eigenvalue $r(M)$ and the eigenvectors $v$ and $u$ are the same whether Eq. (A1.1) is written in $l_{1}\left(F_{k}\right)$ or $l_{1}\left(A^{F_{1}}\right)$. This proves $\sum_{i=1}^{\infty} A^{i} v_{i}<\infty$ and, given the shape of the vector $v$, that for every $R>\frac{\lambda}{A}, \Phi(R)<\infty$. From classical results about power series, $\Phi$ can thus be differentiated near $\lambda$ as: 


$$
\Phi^{\prime}(\lambda)=\sum_{i=1}^{\infty}-i \ell_{i} a_{i} \lambda^{-(i+1)}<\infty
$$

Acknowledgements. We thank the referee for his/her thoughtful recommendations. While writing this paper, Frédéric Gosselin was on leave of absence from the French Institute of Forestry, Agricultural and Environmental Engineering (ENGREF) - 19, Avenue du Maine, 75732 PARIS Cedex 15, FRANCE.

\section{References}

Carey, J. R., Liedo, P., Orozco, D., Vaupel, J. W. 1992. Slowing of mortality rates at older ages in large medfly cohorts. Science 258 (5081), 457-461.

Caswell, H. 2000. Matrix population models: Construction, Analysis, and Interpretation. $2^{\text {nd }}$ Edition. Sinauer, Sunderland, Mass.

Demetrius, L. 1972. On an infinite population matrix. Math. Biosci. 13, 133-137.

Demetrius, L. 1974. Multiplicative processes: II. Mathematical Biosciences 20 (3-4), 345357, doi:10.1016/0025-5564(74)90010-8.

Demetrius, L. 1983. Statistical mechanics and population biology. Journal of Statistical Physics 30 (3), 709-753, doi:10.1007/BF01009685.

Demetrius, L. 1989. Growth rate, population entropy, and perturbation theory. Mathematical Biosciences 93 (2), 159-180, doi:10.1016/0025-5564(89)90021-7.

Ducrocq, V., Besbes, B., Protais, M. 2000. Genetic improvement of laying hens viability using survival analysis. Genetics Selection Evolution 32 (1), 23-40. 
Feller, W. 1966. An introduction to probability theory and its applications. Vol. II. Wiley, New-York.

Feller, W. 1968. An introduction to probability theory and its applications. Vol.1. 3rd edition. Wiley, New-York.

Finch, C. E., 1998. Variations in senescence and longevity include the possibility of negligible senescence. Journals of Gerontology - Series A Biological Sciences and Medical Sciences 53A (4), B235-B239.

Finch, C. E., Austad, S. N., 2001. History and prospects: Symposium on organisms with slow aging. Experimental Gerontology 36, 593-597.

Gaillard, J.-M., Allainé, D., Pontier, D., Yoccoz, N.G., Promislow, D.E.L. 1994. Senescence in natural populations of mammals: a reanalysis. Evolution 48 (2), 509-516.

Gosselin, F. 1997. Modèles stochastiques d'extinction de population: propriétés mathématiques et leurs applications. Unpublished Thesis. Paris 6 University. Paris, France. Gosselin, F., Lebreton, J. D., 2000. The potential of branching processes as a modeling tool for conservation biology, in: Ferson, S., Burgman, M. A. (Eds.), Quantitative methods for conservation biology, Springer, New York, pp. 199-225.

Gosselin, F. 2001. Asymptotic behavior of absorbing Markov chains conditional on nonabsorption for applications in conservation biology. Ann. Appl. Probab. 11 (1), 261-284.

Houllier, F., Lebreton, J.-D. 1986. A renewal equation approach to the dynamics of stagegrouped populations. Math. Biosci. 79 (2), 185-197.

Krein, M.G., Rutman, M.A. 1948. Linear operators leaving invariant a cone in a Banach space. U. Math. Nauk III 3, 3-95, and Amer. Math. Soc. Transl. (1) 10, 199-325.

Lande, R. 1988. Demographic models of the northern spotted owl (Strix occidentalis caurina). Oecologia 75 (4), 601-607., doi:10.1007/BF00776426. 
Lebreton, J.-D. 1996. Demographic models for subdivided populations: the renewal equation approach. Theoret. Popul. Biol. 49 (3), 291-313., doi:10.1006/tpbi.1996.0015.

Lebreton, J.-D., Gosselin, F., Niel, C. 2007. Extinction and viability of populations:

Paradigms and concepts of extinction models. Ecoscience 14 (4), 472-481.

Mertz, D.B. 1971. The mathematical demography of the California Condor population. Am. Naturalist 105, 437-453.

Mueller, L. D., Rose, M. R. 1996. Evolutionary theory predicts late-life mortality plateaus. Proceedings of the National Academy of Sciences of the United States of America 93 (26), 15249-15253, doi:10.1073/pnas.93.26.15249.

Murray, B. G. J. 1991. Comments on the Use of Lotka's Discrete Equations. Oikos 62 (1), $118-122$

Partridge, L., Mangel, M. 1999. Messages from mortality: The evolution of death rates in the old. Trends in Ecology and Evolution 14 (11), 438-442, doi:10.1016/S0169-5347(99)016468.

Pletcher, S. D., Curtsinger, J. W. 1998. Mortality plateaus and the evolution of senescence: Why are old-age mortality rates so low? Evolution 52(2), 454-464.

Rose, M. R., Drapeau, M. D., Yazdi, P. G., Shah, K. H., Moise, D. B., Thakar, R. R., Rauser, C. L., Mueller, L. D. 2002. Evolution of late-life mortality in Drosophila melanogaster. Evolution 56 (10), 1982-1991.

Sasser, D.W. 1964. Quasi-positive operators. Pacific J. Math. 14 (3), 1029-1037.

Seneta, E. 1981. Non negative Matrices and Markov chains. Springer Verlag, Berlin. Steinsaltz, D. 2005. Re-evaluating a test of the heterogeneity explanation for mortality plateaus. Experimental Gerontology 40 (1-2), 101-113., doi:10.1016/j.exger.2004.11.010. 
Styer, L. M., Carey, J. R., Wang, J. L., Scott, T. W. 2007. Mosquitoes do senesce: departure from the paradigm of constant mortality. The American journal of tropical medicine and hygiene $76(1), 111-117$.

Sykes, Z.M. 1969. On discrete stable population theory. Biometrics 25, 285-294.

Usher, M.B. 1972. Development in the Leslie matrix model. In: Jeffers, J.N.T. (Ed.), Mathematical models in Ecology. Blackwell, Oxford, pp.29-60.

Vaupel, J. W., Carey, J. R. 1993. Compositional interpretations of medfly mortality. Science 260 (5114), 1666-1667.

Vaupel, J. W., Carey, J. R., Christensen, K., Johnson, T. E., Yashin, A. I., Holm, N. V., Iachine, I. A., Kannisto, V., Khazaeli, A. A., Liedo, P., Longo, V. D., Zeng, Y., Manton, K. G., Curtsinger, J. W. 1998. Biodemographic trajectories of longevity. Science 280 (5365), 855-860., doi:10.1126/science.280.5365.855.

Vaupel, J. W., Yashin, A. I. 1985. Heterogeneity's ruses: some surprising effects of selection on population dynamics. American Statistician 39 (3), 176-185.

Vere-Jones, D. 1967. Ergodic properties of non-negative matrices, I. Pacific J. Math. 22 (2), 361-386.

Vere-Jones, D. 1968. Ergodic properties of non-negative matrices, II. Pacific J. Math. 26 (3), 601-620. 
Figure 1. Survival rate $b_{i}$ (y axis) as a function of age class $i$ (x-axis) in the model of Example 2. Survival increases from prime age to mature females, and then decreases progressively to reach a senescent plateau of 0.6 , according to $b_{i}=0.6+0.4 \times \frac{1}{1+e^{0.5+0.2 \times i-0.01 \times i^{2}}}$.

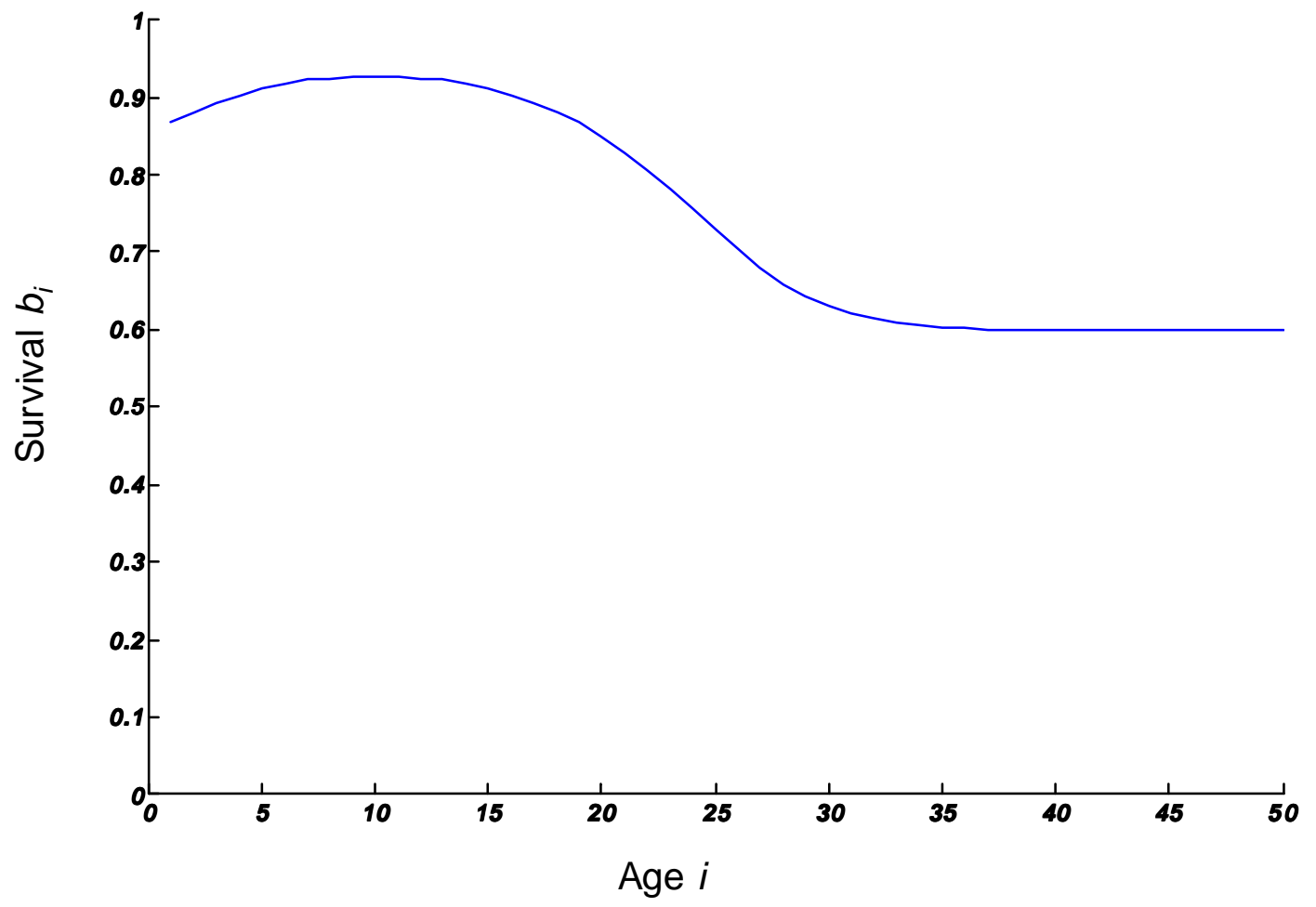


Figure 2. Dominant eigenvalue $\lambda_{n}$ (y-axis) of truncated Leslie (dotted line) and Usher (plain line) matrices as a function of the order of trunctaion $n$ (x-axis).

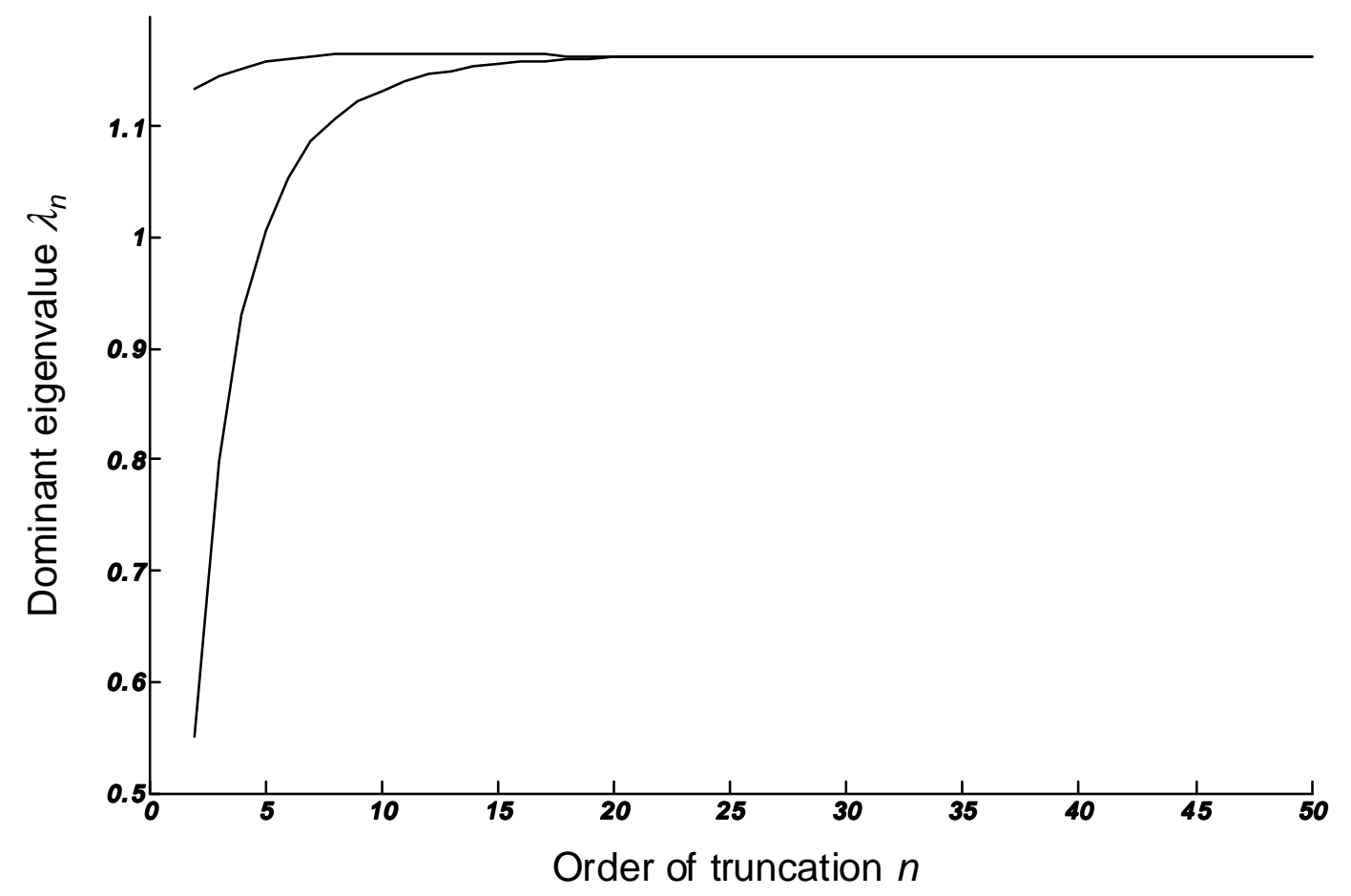

